
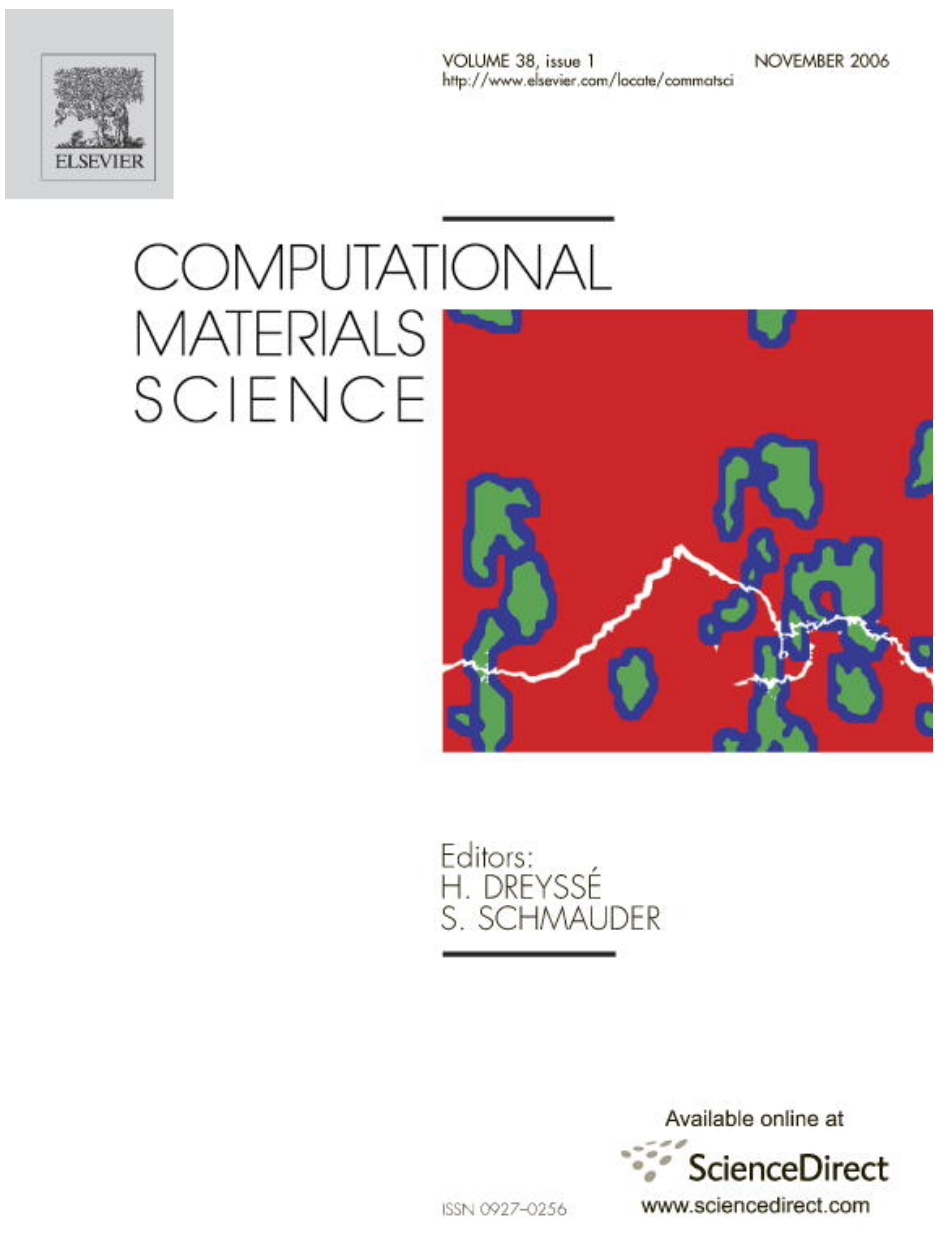

This article was originally published in a journal published by Elsevier, and the attached copy is provided by Elsevier for the author's benefit and for the benefit of the author's institution, for non-commercial research and educational use including without limitation use in instruction at your institution, sending it to specific colleagues that you know, and providing a copy to your institution's administrator.

All other uses, reproduction and distribution, including without limitation commercial reprints, selling or licensing copies or access,

or posting on open internet sites, your personal or institution's website or repository, are prohibited. For exceptions, permission may be sought for such use through Elsevier's permissions site at:

http://www.elsevier.com/locate/permissionusematerial 


\title{
Dislocation patterning: The role of climb in meso-scale simulations
}

\author{
B. Bakó ${ }^{a, *}$, I. Groma ${ }^{\text {a }}$, G. Györgyi ${ }^{a}$, G. Zimányi b \\ ${ }^{a}$ Eötvös University, Institute of Physics, Pázmány Péter sétány 1/A, 1117 Budapest, Hungary \\ ${ }^{\mathrm{b}}$ Department of Physics, University of California, Davis, CA 95616, USA
}

Received 1 December 2005; received in revised form 22 December 2005; accepted 22 December 2005

\begin{abstract}
Simulation of parallel dynamics of edge dislocations in a 2D hexagonal lattice is carried out on a large scale by means of coarse graining, in the absence of external strain. In order to study the effect of climb on dislocation pattern formation, we allowed (i) isotropic (ii) biased (iii) only glide mobility. Moreover we annihilated dislocations with opposite Burgers vectors close to each other. The main result is that in cases (i) and (ii) cellular structure emerges, for (ii) this happens after a longer transient, while (iii) gives a diffuse looking pattern, similar to former single slip plane simulations. In the course of the evolution of dislocation number and cell size they statistically well satisfy Holt's relation. The cell structure found appears to have a characteristic cell size, in contrast to fractal examples in the presence of mechanical strain. Despite the simplicity of our model, the results are consistent with the recently detected low-density dislocation patterns in melt-grown, non-post-deformed crystals.
\end{abstract}

(C) 2006 Elsevier B.V. All rights reserved.

PACS: $61.72 . \mathrm{Bb} ; 61.72 . \mathrm{Lk}$

Keywords: Dislocation patterning; Temperature and climb; Cell structure formation

\section{Introduction}

Revealing the origin of dislocation patterning is a long standing challenge of dislocation theory (see e.g. [1-3] for reviews). During the past three decades several different approaches were proposed to model this self-organization process. An early attempt proposed by Kuhlmann-Wilsdorf et al. [4] is based on the assumption that the dislocation microstructure developing during plastic deformation corresponds to the minimum of the dislocation-dislocation interaction energy. This static approach contradicts the common experimental observations that dislocation structures strongly depend on the mode and the rate of deformation. In the early 80 s, applying the concept proposed to model oscillating chemical reactions, Walgraef and Aifantis [5,6] suggested a reaction-diffusion model to

\footnotetext{
* Corresponding author. Tel.: +36 12090555x6457; fax: +36 13722811. E-mail address: botond.bako@metal.elte.hu (B. Bakó).
}

account for the development of spatial fluctuations of dislocation density. Although the model is able to predict several features of dislocation microstructure, in the evolution equations proposed the physical origin of the different terms are not really justified so far. Based on specific dislocation mechanisms, Kratochvil et al. [2,7] developed a continuum model that is also able to predict periodic dislocation patterning. However, some of the details of the model are not completely developed. A recent promising approach is to apply statistical methods to treat the collective behavior of large dislocation ensembles [8-13]. Up to now, however, these investigations have not lead to conclusive results on dislocation pattern formation.

It can be stated that at the moment there is no generally accepted theory of dislocation patterning. According to our opinion the main difficulty is that it is not clear what are the really important phenomena among the many possible ones which play a important role in the patterning process. There are indications that under certain circumstances the long range dislocation-dislocation interaction 
is enough to obtain cell like dislocation structures [14]. On the other hand it is quite unlikely that for example the short range dislocation-dislocation interaction leading to junction formation does not have an influence on patterning. An important recent experimental observation by Rudolph et al. [15] is that the dislocation climb can strongly change the evolving dislocation pattern morphology. By studying the dislocation structure developing during the crystal growth process of GaAs single crystals they have found that if the material composition and other experimental conditions are set up so that the dislocation climb is negligible compared to glide the dislocation configuration remains nearly random. If, however, dislocation climb is "turned on" a cell-like dislocation structure develops with a characteristic cell size even in the absence of post-grown deformation. The role of climb was emphasized long ago by Blum [16]. This problem, however was not addressed in the theoretical models mentioned above $[5,6,2,7,13]$ They correspond to low-temperature dipolar patterning.

Discrete dislocation dynamics (DDD) simulation is an ideal tool to study the influence of different type of interactions and different microscopic dislocation phenomena on patterning. In simulations these parameters can be "turned on" or "off" to see their role. So, DDD simulations can help to resolve the above mentioned problems.

To investigate realistic cases one should carry out $3 \mathrm{D}$ simulations. At the moment, however, due to the complexity of the problem, the size of the simulation volume, and the dislocation density affordable they are not enough to obtain statistically relevant information about well developed dislocation patterns [17-20]. Although 2D simulation models are strong simplifications of the real dislocation systems, they can provide results of physical relevance [21-24].

In the present paper the role of dislocation climb in dislocation patterning is investigated. The work is motivated by the recent experimental results of Rudolph et al. [15]. We set up a simulation method based on a model 2D system which allows us to study the collective behavior of up to 40000 dislocations. Similar investigations were performed earlier by Argaman et al. [24], but on a much smaller systems (few hundred dislocations). The dislocation number used in our studies makes possible to identify the "macroscopic" characteristics of the dislocation structures developing.

\section{Numerical method}

Motivated by the slip geometry of the triangular lattice structure formed e.g. by the vortices in type II superconductors, in the simulations reported a 2D system of edge dislocations with three different possible Burgers vectors defined by the directions $\pm(\cos (m \pi / 3), \sin (m \pi / 3)), \quad m=$ $1,2,3$ in a square area of size $L \times L$ was considered. In order to avoid surface effects, periodic boundary condition was used. In this construction the two glide directions, with angles $\pm \pi / 3$ with the $x$ axis, have irrational winding num- ber in the toroidal square. Therefore, when those axes cross the square boundary, they do not close. This results in a more random sequence of configurations, thus it helps to randomize the simulation ensemble. Since it turned out that dislocations rarely traveled long distances, this feature had little significance.

When we start to set up a numerical schema to determine the time evolution of the dislocation system we immediately face to the problem that the calculation of the stress components created by an individual dislocation with Burgers vector $\left(b_{x}, b_{y}\right)$ placed in the center of the simulation area, with periodic boundary conditions is a non-trivial task. It cannot be solved analytically.

In order to treat the problem, the formalism developed by Kröner [25], commonly called as "continuum theory of dislocations" is used. According to this theory for a straight dislocation placed in the origin, extending parallel to the $z$ direction the Airy's function $\chi(r)$ fulfill the biharmonic equation

$\nabla^{4} \chi=C\left(b_{x} \frac{\partial}{\partial y}-b_{y} \frac{\partial}{\partial x}\right) \delta(x) \delta(y)$

where $C$ is a combination of elastic moduli. For 3D planar problems (i.e. when the problem has a translation invariance in a certain direction) $C \equiv \frac{2 \mu}{1-v}$, while for real 2D crystals, like e.g. the Abrikosov vortex lattice in thin film superconductors $C \equiv 2 \mu(1+v)$, in which $\mu$ is the shear modulus and $v$ is the Poisson's ratio. From $\chi(r)$ the stress components read as

$\sigma_{x x}=-\frac{\partial^{2} \chi}{\partial y^{2}}, \quad \sigma_{y y}=-\frac{\partial^{2} \chi}{\partial x^{2}}, \quad$ and $\quad \sigma_{x y}=\frac{\partial^{2} \chi}{\partial x \partial y}$.

The internal force $\vec{F}$ acting on a unit length part of a dislocation with Burgers vector $\vec{b}$ in an elastic field can be given by the Peach-Koehler formula

$\vec{F}=(\hat{\sigma} \vec{b}) \times \vec{l}$

in which $\vec{l}=(0,0,1)$ and the stress tensor $\sigma$ is the sum of stress components created by the individual dislocations and the external stress.

The field equation (1) with periodic boundary conditions can be relatively easily solved in the Fourier space [26]. According to the relations in (2), knowing $\chi(r)$ the stress components can also be easily calculated. A common approach for solving Eqs. (1) and (2) is to introduce the discretized differential operators in the Fourier space. By considering $M$ mesh points both in the $x$ and $y$ directions, the first order discretized operators corresponding to $\partial / \partial x$, $\partial / \partial y, \partial^{2} / \partial x^{2}$, and to $\partial^{2} / \partial y^{2}$ can be given as

$D_{m}=\mathrm{i} \frac{M}{L} \sin \left(\frac{2 \pi}{M} m\right)$

$D_{m}^{2}=2 \frac{M^{2}}{L^{2}}\left[\cos \left(\frac{2 \pi}{M} m\right)-1\right]$

where $m=\overline{0, M-1}$ denotes the indices of the mesh points in the $x$ and $y$ directions. 
By Fourier transforming Eqs. (1) and (2) and using the operators defined by (4) and (5), the Fourier transform of the stress components read as

$$
\begin{aligned}
& \tilde{\sigma}_{x x}(l, m)=-C D_{l} D_{l} \frac{b_{x} D_{m}-b_{y} D_{l}}{\left[D_{l}^{2}+D_{m}^{2}\right]^{2}}, \\
& \tilde{\sigma}_{y y}(l, m)=-C D_{m} D_{m} \frac{b_{x} D_{m}-b_{y} D_{l}}{\left[D_{l}^{2}+D_{m}^{2}\right]^{2}}, \\
& \tilde{\sigma}_{x y}(l, m)=C D_{l} D_{m} \frac{b_{x} D_{m}-b_{y} D_{l}}{\left[D_{l}^{2}+D_{m}^{2}\right]^{2}} .
\end{aligned}
$$

The inverse discrete Fourier transforms of the above expressions give the corresponding stress components at the grid points. Certainly if two dislocations are closer to each other than the grid distance the approximate stresses obtained by the method explained above cannot be applied to calculate the interaction force between the two dislocations. In order to solve this problem in this case the stress expressions

$\sigma_{x x}^{\text {ind }}=\frac{C}{4 \pi} \frac{b_{y} x\left(x^{2}-y^{2}\right)-b_{x} y\left(3 x^{2}+y^{2}\right)}{\left(x^{2}+y^{2}\right)^{2}}$,

$\sigma_{y y}^{\text {ind }}=\frac{C}{4 \pi} \frac{b_{x} y\left(x^{2}-y^{2}\right)+b_{y} x\left(x^{2}+3 y^{2}\right)}{\left(x^{2}+y^{2}\right)^{2}}$,

$\sigma_{x y}^{\text {ind }}=\frac{C}{4 \pi} \frac{\left(b_{x} x+b_{y} y\right)\left(x^{2}-y^{2}\right)}{\left(x^{2}+y^{2}\right)^{2}}$,

corresponding to an infinite body can be used. On the other hand, if two dislocations are not in the same box defined by the grid, with the precision determined by the number of mesh points $M$ introduced, their interaction force is insensitive to their actual position within their "own" box. This results that at a given dislocation the stress contribution, coming from the dislocations which are not in the same box as the one considered, can be calculated as a convolution of the Fourier transform of stress expression given by Eq. (6) and a coarse-grained dislocation Burgers vector density field $\alpha_{i}(l, m)$ that is the net Burgers vector averaged for the box with indices $l$ and $m$. So the total stress acting on the $i$ th dislocation being in the box indexed by $(l, m)$ is

$\hat{\sigma}\left(\vec{r}_{i}\right)=\hat{\sigma}^{s f}(l, m)+\sum_{j} \hat{\sigma}^{\text {ind }}\left(\vec{r}_{i}-\vec{r}_{j}\right)$,

in which the sum needs to be performed only for those dislocations which are in the same box as the $i$ th dislocation, and $\hat{\sigma}^{s f}(l, m)$ is the Fourier transform of

$$
\begin{aligned}
& \tilde{\sigma}_{x x}^{s f}(l, m)=C D_{l} D_{l} \frac{\tilde{\alpha}_{y}(l, m) D_{l}-\tilde{\alpha}_{x}(l, m) D_{m}}{\left[D_{l}^{2}+D_{m}^{2}\right]^{2}}, \\
& \tilde{\sigma}_{y y}^{s f}(l, m)=C D_{m} D_{m} \frac{\tilde{\alpha}_{y}(l, m) D_{l}-\tilde{\alpha}_{x}(l, m) D_{m}}{\left[D_{l}^{2}+D_{m}^{2}\right]^{2}}, \\
& \tilde{\sigma}_{x y}^{s f}(l, m)=C D_{l} D_{m} \frac{\tilde{\alpha}_{x}(l, m) D_{m}-\tilde{\alpha}_{y}(l, m) D_{l}}{\left[D_{l}^{2}+D_{m}^{2}\right]^{2}} .
\end{aligned}
$$

where $\tilde{\alpha}_{i}(l, m)$ is the Fourier transform of the coarsegrained dislocation Burgers vector field $\alpha_{i}(l, m)$.
The stress calculation method explained above can be considered as a special Fast Multipole Expansion Method. According to our experience up to about 40000 dislocations and $256 \times 256$ grid points this method can be very efficiently applied. It is about 10 times faster than the direct calculation of the pair interactions.

In most of the discrete dislocation dynamics simulations it is assumed that the glide component of the friction force (accounting for the dissipative nature of dislocation motion) acting on the dislocation is proportional the glide velocity (see e.g. [27-29]). Beside this, in most cases the inertia term can be neglected beside the friction force (over-damped assumption). With these assumptions the glide velocity of the $i$ th dislocation is

$\vec{v}_{\mathrm{g}}^{i}=\Gamma_{\mathrm{g}} \frac{\left(\vec{F}^{i} \vec{b}^{i}\right)}{b} \frac{\vec{b}^{i}}{b}$

where $\Gamma_{\mathrm{g}}$ is the glide mobility and $\vec{F}^{i}$ is the Peach-Koehler force given by Eq. (3).

Since the purpose of the present paper is to investigate the role of dislocation climb, we have to set up rule for the dislocation velocity in the direction perpendicular to the Burgers vector of the dislocations. The dislocation climb is a stress assisted thermally activated process with a preferred jumping probability in the direction of the "climb component" of the Peach-Koehler force $F_{\mathrm{c}}^{i}=\vec{F}^{i} \vec{n}^{i}$ where $\vec{n}^{i}$ is an unit vector perpendicular to the Burgers vector of the dislocation considered. More precisely, stress assisted thermally activated process means that the jumping probability in the "forward" direction is proportional to $\exp \left[-\left(E-a^{2} F_{\mathrm{c}}\right) / k T\right]$ where $E$ is the activation energy of jumping without stress, $a$ is a constant with length dimension, $k$ is the Boltzmann constant, and $T$ is the temperature. On the other hand the jumping probability in the "backward" direction is proportional to $\exp \left[-\left(E+a^{2} F_{\mathrm{c}}\right) /\right.$ $k T]$. It is easy to see from these expressions that the mean value of the dislocation velocity in the climb direction is proportional to $F_{\mathrm{c}}$, if $F_{\mathrm{c}}$ is small enough. So, by neglecting the thermal fluctuation, the climb velocity can be given as

$\vec{v}_{\mathrm{c}}^{i}=\Gamma_{\mathrm{c}}\left(\vec{F}^{i} \vec{n}^{i}\right) \vec{n}^{i}$

where $\Gamma_{\mathrm{c}}$ represent the climb mobility. The same approximation was used earlier by Argaman et al. [24,30]. (It needs to be mentioned that similar argument holds for glide motion with different parameters.)

As our main aim is to demonstrate the effect of climb on dislocation patterning we present the two extremal situations of $\Gamma_{\mathrm{c}}=\Gamma_{\mathrm{g}}$ (isotropic mobility) and $\Gamma_{\mathrm{c}}=0$ (no climb). In our simulations annihilation of dislocations was allowed, namely, when a pair of them on the same glide axis but of opposite signs reached closer to each other than the grid distance they were taken out of the system. Since our simulation intends to mimic dislocation motion in asgrown crystals, so we do not apply external mechanical stress, only stress exerted on a dislocation by the others is included. As a consequence of this, dislocation multiplication was not introduced. 


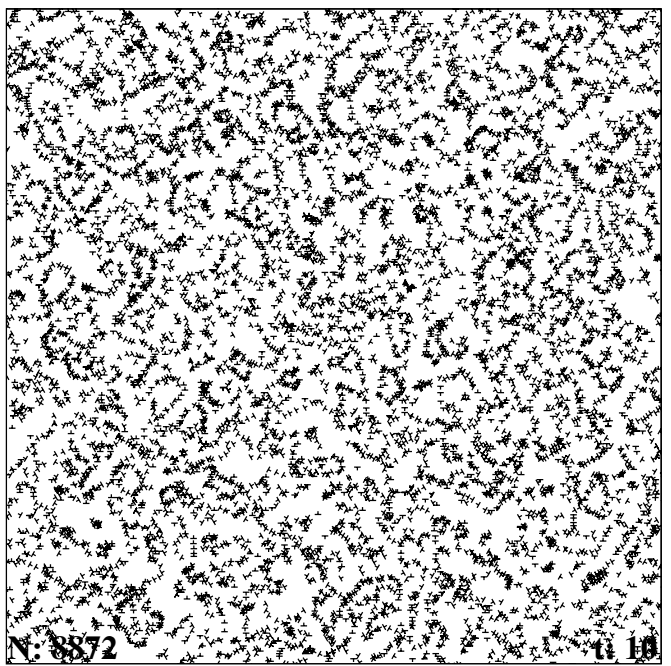

(a)

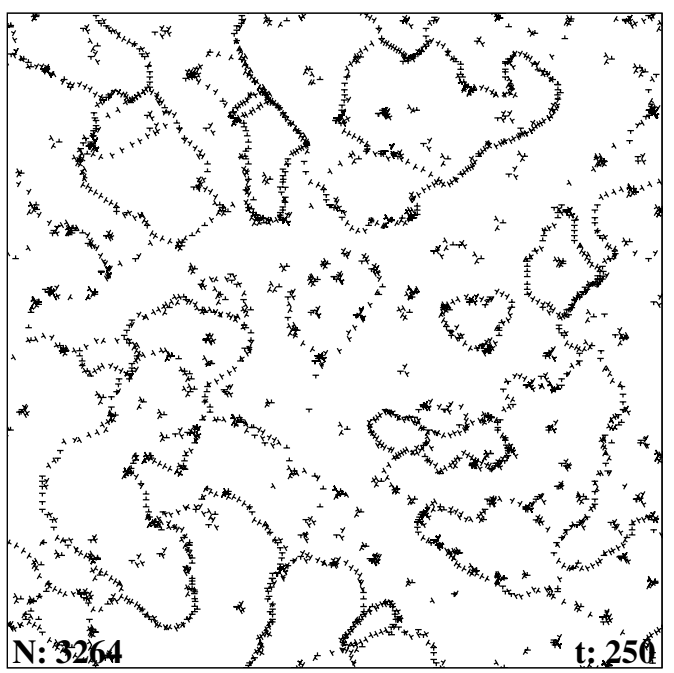

(c)

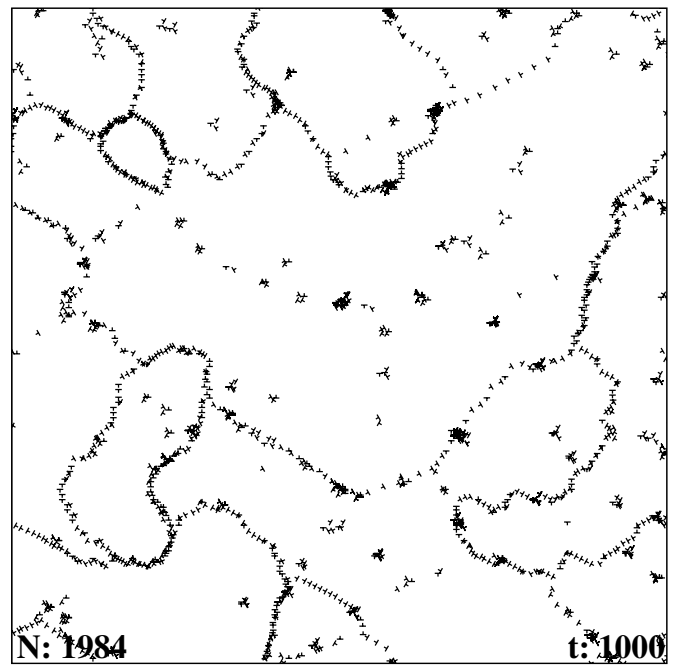

(e)

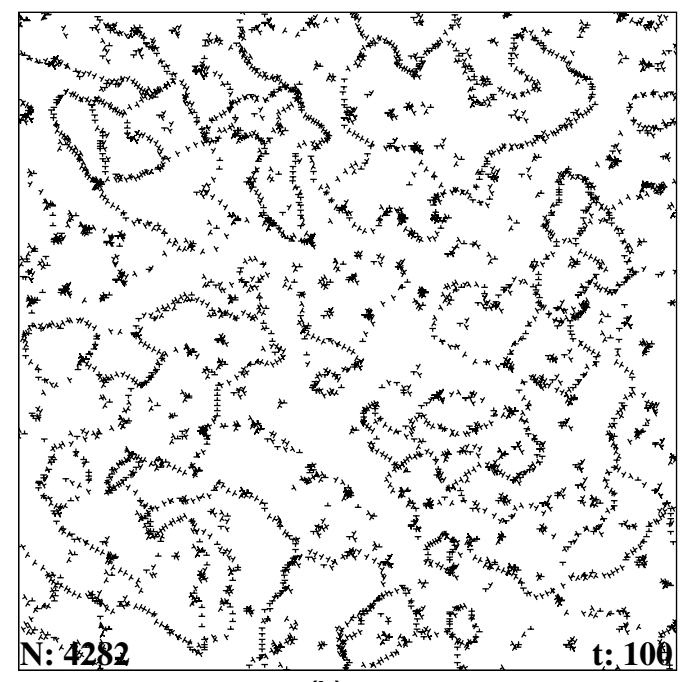

(b)

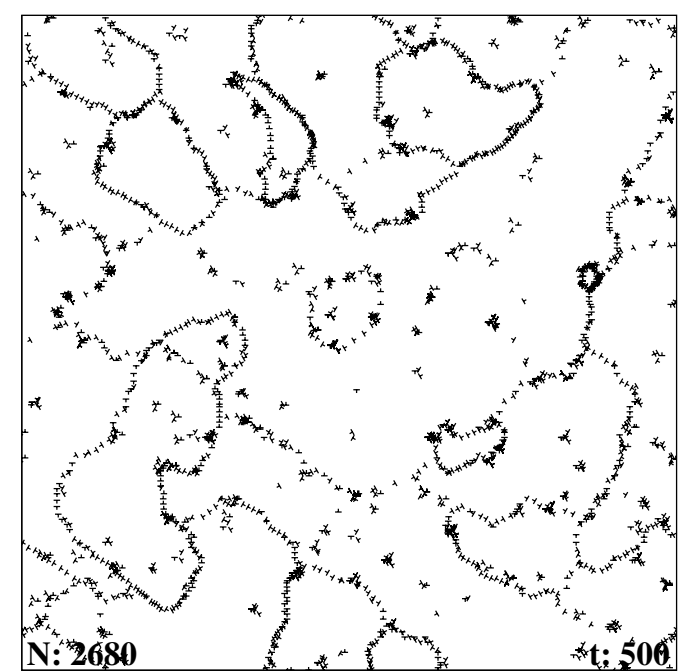

(d)

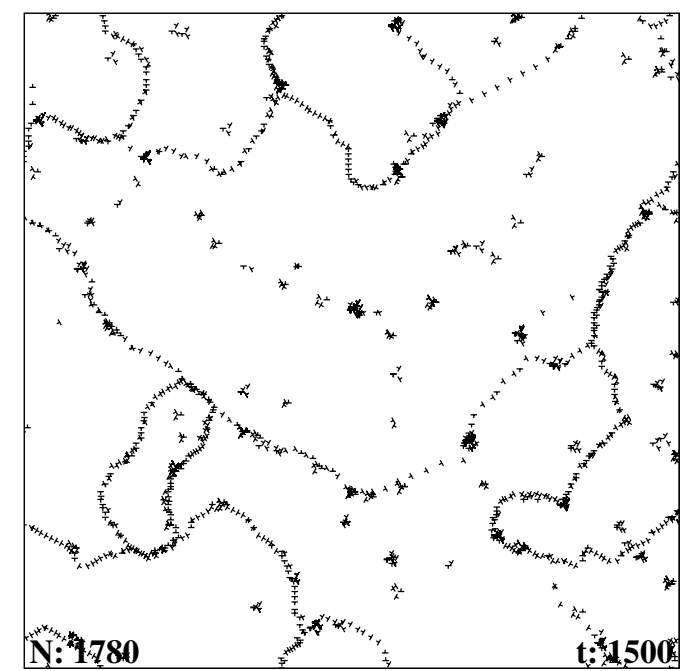

(f)

Fig. 1. Time evolution of dislocation configurations of the simulation discussed in the text, corresponding to $\Gamma_{\mathrm{c}}=\Gamma_{\mathrm{g}}$. The actual dislocation number for each snapshot is shown in its bottom left corner, while the elapsed time is displayed in the right bottom corner. 
Without restricting the generality we can set $L=1$, meaning the distances are measured in simulation box size unit. On the other hand the glide mobility $\Gamma_{\mathrm{g}}$ can also be scaled out by introducing time unit in which $\Gamma_{\mathrm{g}} b C /$ $4 \pi L^{2}=1$. So, the behavior of the system is determined only by the number of the dislocations in the different slip systems and the $\Gamma_{\mathrm{c}} / \Gamma_{\mathrm{g}}$ ratio.

In the simulations reported here $256 \times 256$ grid points were introduced. A couple of simulations were performed with larger number of grid points, but according to our experience the overall response of the system remained the same. We tested the role of the time step $\mathrm{d} t$ by reducing it so that numerical error was sufficiently suppressed; we found $\mathrm{d} t=2 \times 10^{-8}$ satisfactorily small.

\section{Simulation results}

First we study the isotropic case, $\Gamma_{\mathrm{c}}=\Gamma_{\mathrm{g}}$. Starting out from a random configuration of initially $N=40000$ dislocations we let the system relax. Fig. 1 shows snapshots for subsequent time slices. It is apparent, that after a relatively short transient a cell like pattern emerges with dislocations accumulating in walls of various linear densities. Most of the walls are quite sharp, containing dislocations of the same sign, aligned in one of the six main directions thus creating polygonal structures. They are small angle grain boundaries. In the course of time, the boundaries slowly deform, move, and some of them dissolve, while due to dipole annihilation the total number of dislocations keeps decreasing.

Dislocation cell formation was already noted in the simulations of Argaman et al. [24,30], where a single dislocation feeling the largest force of all, was moved in a time step. Here the coarse-graining of the distribution of dislocations allowed us to apply a more faithful parallel dynamics as well as a much larger ensemble. It also made us possible to follow further the merging of cells from the dislocation-rich configuration of early times to the rarefied

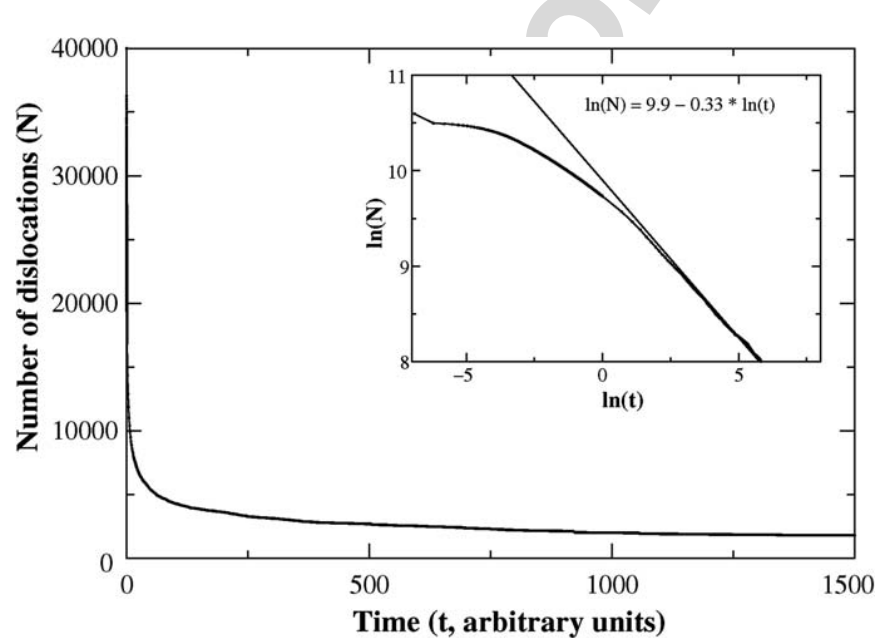

Fig. 2. Time evolution of the dislocation number $N$ for the system shown in Fig. 1.

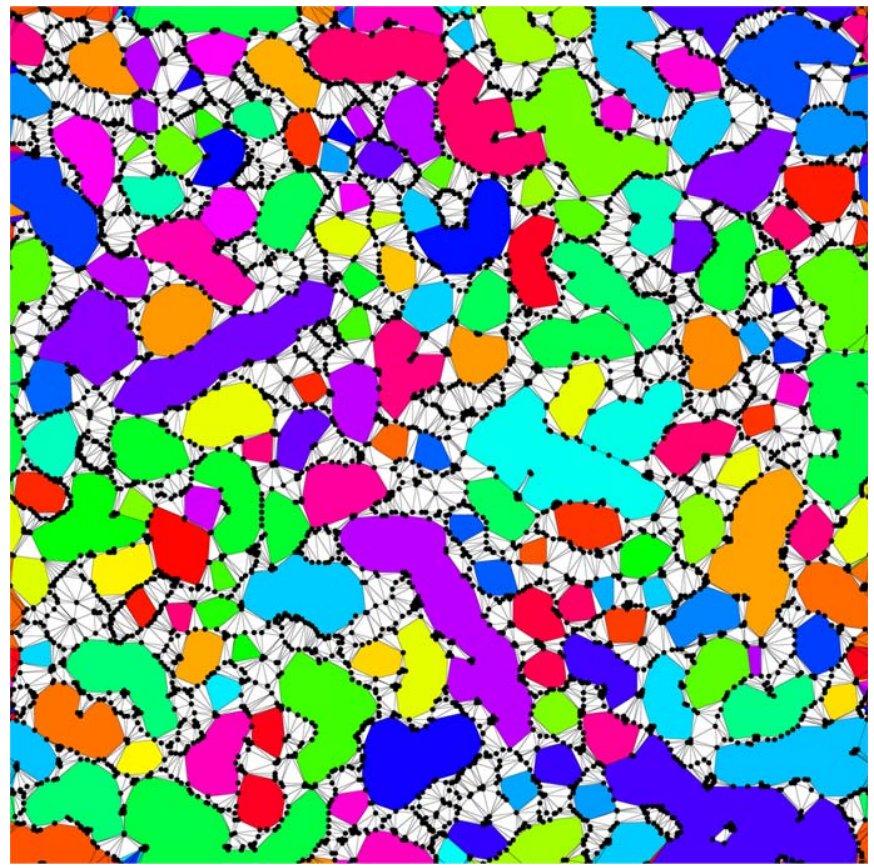

Fig. 3. Cell structure at $t=50$ obtained by clipping the Delaunay triangular lattice of long edges.

picture of larger-scale structures. In addition, our study includes moderate and extreme anisotropy. We should note that $[24,30]$ contains a nice analysis of data on deformation of the underlying lattice due to dislocations, a direction we do not discuss here but relegate to later work.

In order to quantify the apparent growth of cell size with time, we first present the time evolution of the dislocation number, as shown in Fig. 2. We find that the number of dislocations decays according to the power law

$N \propto t^{-1 / 3}$.

Note that the exponent was obtained only numerically, and to this date we are not aware of any theory accounting for it. As a further step, we apply a geometrical definition of a cell as follows. For a given dislocation configuration we performed Delaunay triangulation [31]. Then removing (clipping) all links longer than a certain value $d_{\max }$ depending on the dislocation density yields a graph wherein the contiguous areas enclosed by loops are identified as cells. (The actual value of $d_{\max }$ was determined "empirically". In the simulations reported $d_{\max }=200 / N$ was used.) A typical cell structure of the clipped Delaunay lattice is shown in Fig. 3.

In the course of dislocation annihilation cells merge. According to Holt's relation [32], the characteristic linear size of cells is

$d=K N^{-1 / 2}$

and since the mean cell area $S \propto d^{2}$, we expect

$S \propto N^{-1}$.

As it can be seen in Fig. 4, the Holt's relation is fulfilled. 


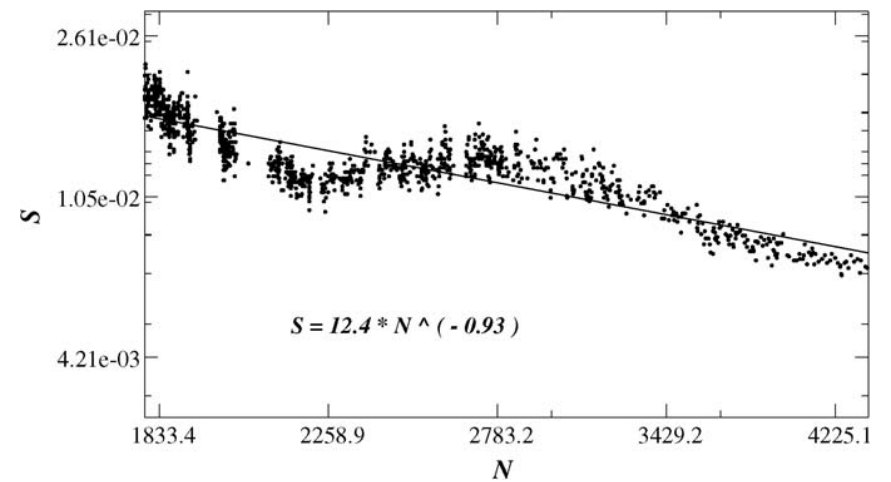

Fig. 4. Mean cell area $S$ dependence as function of the number of dislocations. Since $S \propto d^{2}$, Holt's relation is satisfied.

It should be mentioned that the Delaunay triangulation resulted in small triangular "cells" formed only by three dislocations (see white triangles in Fig. 3). Since they obviously cannot be considered as real cells, they were not taken into account in the cell size statistics.

Dislocation mobility is of course not isotropic for real crystals. In order to gain a rough picture of the effect of moderate anisotropy, we considered the case $\Gamma_{\mathrm{c}}=$ $0.1 \cdot \Gamma_{\mathrm{g}}$. Here we observed longer transients but later granular patterns emerged similar to the ones presented above.

The case of strong anisotropy is represented by $\Gamma_{\mathrm{c}}=0$. Simulations with only glide motion were performed before on large scales with and without external stress (see [2729,1,33-39]). Since dislocations are confined to their glide axes, annihilation in the long run is practically negligible,

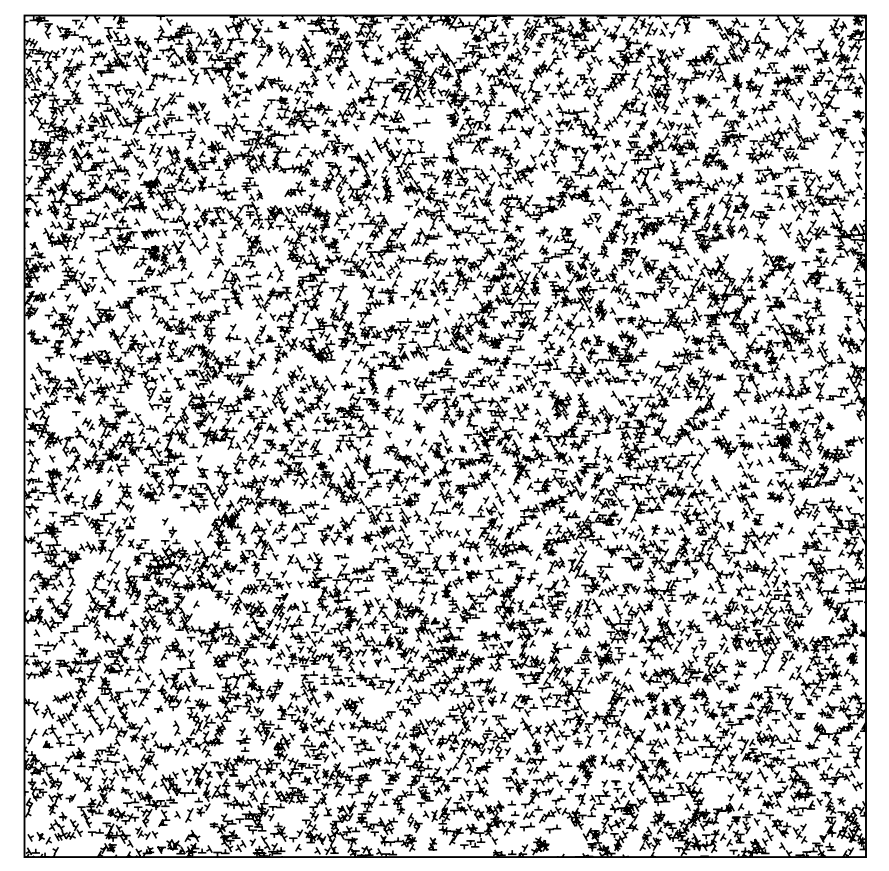

Fig. 5. Typical dislocation structure obtained in the absence of climb for $t=1500$. and in the absence of external stress the initially randomly placed dislocations relax towards some equally random looking "diffuse" configuration. To demonstrate the visual difference to the cell structure we repeated our simulation without climb and present the result in Fig. 5.

\section{Discussion and comparison with experiments}

The literature on dislocation pattern formation is vast (see e.g. $[1,33]$ ). Recent experimental accounts by Rudolph et al. [15] and Rudolph [40] focusing on dislocation patterns display characteristic structures, which are worth being compared qualitatively to our results. In melt-grown undoped GaAs crystals dislocations generated by thermal induced strain, but in the absence of post-grown mechanical stress, were visualized on sections by the etch-pit technique as shown in Fig. 6 taken from [15]. The authors were able to control stoichiometry in situ by vapor atmosphere about the melt and produced wafers of both

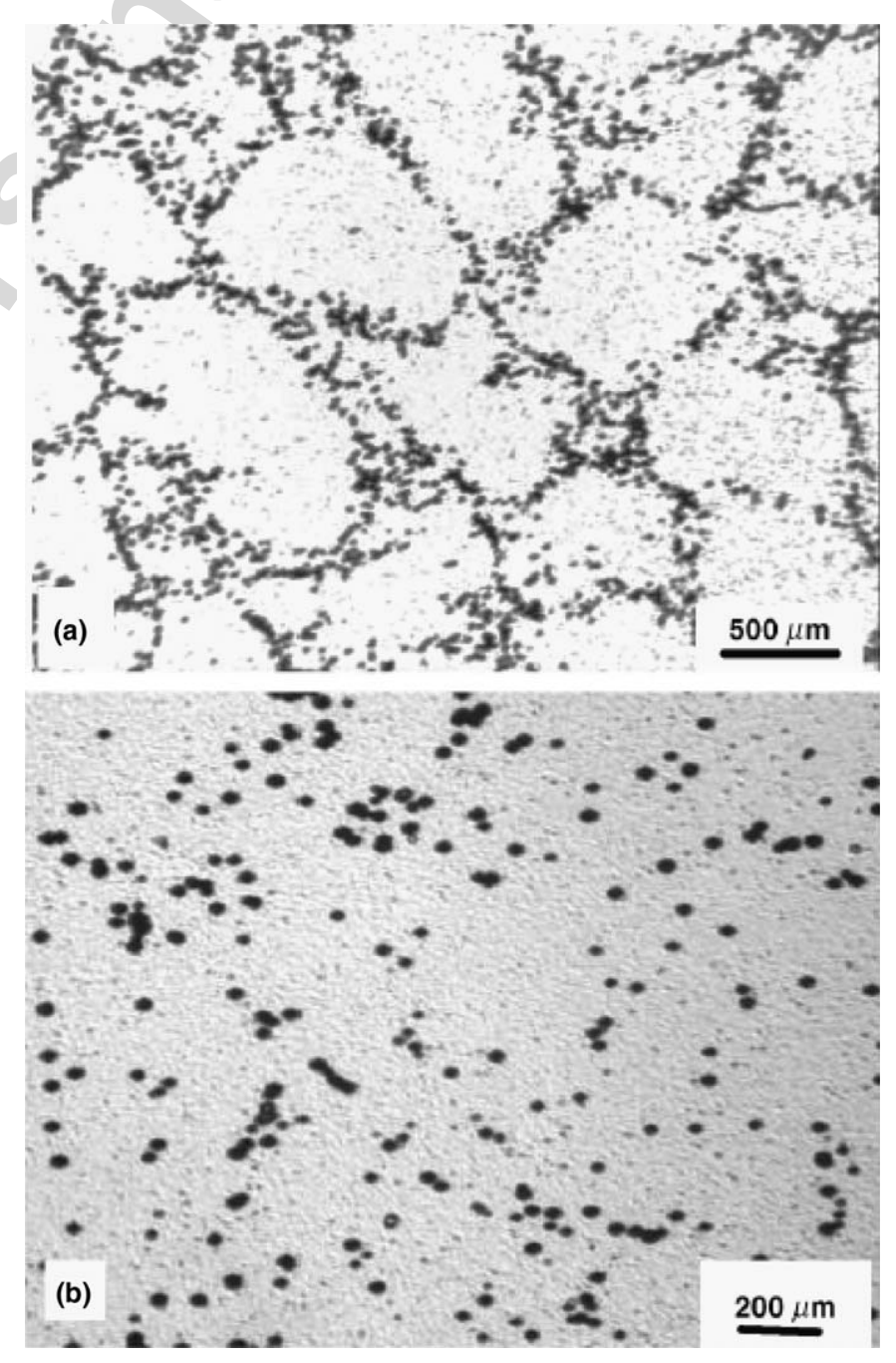

Fig. 6. Etch-pit distributions from Rudolph et al. [15]. (a) Well-developed cellular structure typical for off-stoichiometric GaAs crystals. (b) Diffuse configuration in near-stoichiometric GaAs crystal. 
near-stoichiometric and off-stoichiometric compositions. They attribute the appearance of cells on picture (a) of Fig. 6 to enhanced climb due to the point defects caused by off-stoichiometry, while suggest that climb may be suppressed in a more perfect crystal, wherein they find the "diffuse" dislocation distribution of (b). They also test Holt's relation which is satisfied within experimental error. In our simulation, when climb was important, we obtained with annihilation a cleaner granular structure (Fig. 1). None of our simulation patterns can be mistaken for the real ones, mostly because in our case sharper walls appear. However, we neglected entropic effects that may arise at high temperature of melt-growth and can contribute to widening of walls. Furthermore, we did not fine-tune annihilation, but either allowed a large annihilation distance. And, of course, we only simulated 2D systems, while the experimental evidences are $2 \mathrm{D}$ sections of $3 \mathrm{D}$ crystals. It should be stressed that also sharp walls of single dislocation lines are observed in other growth processes, as in telluride crystals [40], similar to the numerically obtained wall structure. There are, however, numerous stray dislocations within cells in [40], while on our Fig. 1 we see less randomly placed lone dislocations.

On the other hand, when climb was switched off, the diffuse nature of the pattern is recovered here as in Fig. 5, similarly to earlier, smaller scale simulations [41]. It has been emphasized in [40] that such structures can also be observed in case of doping beyond a threshold, and attributed it to the effect of some dopant as mobility stoppers. Indeed, if slow mobility in any direction greatly increases the time needed to reach some stationary configuration, it may prevent cell formation under a given experimental condition.

\section{Conclusion}

While in real crystals dislocation structures can be of a variety of types, the two main patterns of granular and diffuse distributions can be discerned. Here we presented simulation results highlighting the two extremes, cells emerging when mobility was bidirectional, and randomlooking point distributions when only glide was allowed. We concentrated on the case without external, mechanical strain, and found that the cells are of a characteristic size nearly satisfying Holt's relation.

\section{Acknowledgements}

Financial support of the European Community's Human Potential Programme under Contract no. MRTNCT-2003-504634 [SizeDepEn] and the Hungarian Scientific Research Fund (OTKA) under Contract nos. T 043519 and TS 044839 is gratefully acknowledged.

\section{References}

[1] L. Kubin, Dislocation patterning, in: R. Cahn, P. Haasen, E. Kramer (Eds.), Material Science and Technology: A Comprehensive Treatment, vol. 6, VCH, Weinheim, 1993, p. 137.

[2] J. Kratochvil, Solid State Phenom. 35-36 (1994) 71.

[3] R. Amodeo, N. Ghoniem, Phys. Rev. B 41 (1990) 6958.

[4] D. Kuhlmann-Wilsdorf, J. van der Merve, Mater. Sci. Eng. 55 (1982) 79.

[5] D. Walgraef, E. Aifantis, Int. J. Eng. Sci. 23 (1985) 1351.

[6] D. Walgraef, E. Aifantis, Res. Mech. 23 (1988) 161.

[7] L. Kubin, J. Kratochvil, Philos. Mag. A 80 (1) (2000) 201.

[8] I. Groma, Phys. Rev. B 52 (1997) 8025.

[9] I. Groma, B. Bakó, Phys. Rev. B 58 (1998) 2969.

[10] B. Bakó, I. Groma, Phys. Rev. B 60 (1999) 122.

[11] A. El-Azab, Phys. Rev. B 61 (2000) 11956.

[12] R. Sedlacek, J. Kratochvil, E. Werner, Philos. Mag. 83 (2003) 3735.

[13] J. Kratochvil, R. Sedlacek, Phys. Rev. B 67 (2003) 094105.

[14] I. Groma, B. Bakó, Phys. Rev. Lett. 84 (2000) 1487.

[15] P. Rudolph, C. Frank-Rotsch, U. Juda, F.-M. Kiessling, Mater. Sci. Eng. A 400-401 (2005) 170.

[16] W. Blum, High-temperature deformation and creep of crystalline solids, in: R. Cahn, P. Haasen, E. Kramer (Eds.), Material Science and Technology: A Comprehensive Treatment, vol. 6, VCH, Weinheim, 1993, pp. 359-405.

[17] V. Bulatov, M. Tang, H. Zbib, MRS Bull. 26 (2001) 195

[18] B. Devincre, L. Kubin, C. Lemarchand, R. Madec, Mater. Sci. Eng. A $309-310$ (2001) 211.

[19] X. Han, N. Ghoniem, Z. Wang, Philos. Mag. 83 (2003) 3705.

[20] K. Schwarz, Phys. Rev. Lett. 78 (1997) 4785.

[21] H. Cleveringa, E. van der Giessen, A. Needleman, Int. J. Plast. 15 (1999) 837.

[22] H. Cleveringa, E. van der Giessen, A. Needleman, Philos. Mag. A 79 (1999) 863.

[23] A. Benzerga, Y. Brechet, A. Needleman, E.V. der Giessen, Model. Simul. Mater. Sci. Eng. 12 (2004) 159.

[24] N. Argaman, O. Levy, G. Makov, Mater. Sci. Eng. A 309-310 (2001) 386.

[25] E. Kröner, Continuum theory of defects, in: R. Balian et al. (Eds.), Physics of Defects, Les Houches Session XXXV, 1980, NorthHolland, Amsterdam, 1981.

[26] H. Nussbaumer, in: Fast Fourier Transform and Convolution Algorithms, Springer-Verlag, New York, 1982.

[27] I. Groma, G. Pawley, Philos. Mag. A 67 (1993) 1459.

[28] A. Gullouglu, D. Srolovitz, R. LeSar, P. Lomdahl, Scripta Metall. 23 (1989) 1347.

[29] A. Gullouglu, C. Hartley, Mod. Simul. Mater. Sci. Eng. 1 (1992) 1.

[30] N. Argaman, O. Levy, G. Makov, J. Phys. IV 11 (2001) 61.

[31] A. Okabe, B. Boots, K. Sugihara, in: Spatial Tessellations: Concepts and Applications of Voronoi Diagrams, Wiley, New York, 1992, p. 94.

[32] D. Holt, J. Appl. Phys. 41 (1970) 3197.

[33] L. Kubin, in: A. Goniss, P. Turchi (Eds.), Dislocation Patterns: Experiment, Theory and Simulation, NATO ASI on Stability of Materials, Plenum, Corfu, 1996.

[34] R. Amodeo, N. Ghoniem, Phys. Rev. B 41 (1989) 6958.

[35] R. Amodeo, N. Ghoniem, Res. Mech. 23 (1988) 137.

[36] A. Gullouglu, C. Hartley, Mod. Simul. Mater. Sci. Eng. 1 (1992) 383.

[37] V. Lubarda, J. Blume, A. Needleman, Acta. Metall. Mater. 41 (1993) 625.

[38] J. Hirth, M. Rhee, H. Zbib, J. Comput. Aided Des. 3 (1996) 164.

[39] H. Cleveringa, E. van der Giessen, A. Needleman, Acta Mater. 45 (1997) 3163.

[40] P. Rudolph, Cryst. Res. Technol. 40 (1/2) (2005) 7.

[41] F. Csikor, I. Groma, Phys. Rev. B 70 (2004) 064106. 